\title{
Lumbar transcutaneous electrical nerve stimulation to improve exercise performance in COPD patients
}

\begin{abstract}
To the Editor:
Muscle group III (A $\delta$ fibres) and IV (C fibres) sensory afferents are involved in the cardiorespiratory adaptation to exercise $[1,2]$. Their inhibition with intrathecal fentanyl in the dorsal horn of the spinal cord to block their cortical projections decreases high-intensity constant workload endurance performance in healthy athlete subjects because of a blunted cardiorespiratory response to exercise. In this condition with high metabolic demand, any decrease in ventilation or haemodynamics would compromise performance because of a nearly maximal solicitation without any possibility for a compensatory strategy. In contrast, GAGNON et al. [3] have published that the use of spinal anaesthesia with fentanyl with the goal of inhibiting muscle group III and IV fibres in chronic obstructive pulmonary disease (COPD) patients improved dyspnoea and endurance capacity. This improvement was due to the blunted ventilatory response to exercise which improved physiological dead space, ventilatory efficiency and in turn, dyspnoea. Moreover, at this relatively lower external workload compared with healthy subjects, cardiac output and peripheral oxygen extraction were not maximal and any mitigation in cardiac output (if any) would be overcome by an increase in peripheral muscle oxygen extraction [3]. High-frequency or low-frequency transcutaneous electrical nerve stimulation (TENS) provide a less invasive alternative which activates opioid receptors, especially those located in the dorsal horn of the spinal cord $[1,4,5]$. This approach deserves to be studied during exercise and over a course of pulmonary rehabilitation in these patients. We performed a randomised double-blind study (clinicaltrials.gov NCT03312322) to assess whether high-frequency or low-frequency lumbar TENS could improve endurance exercise capacity in patients with COPD. Secondary objectives were to assess the influence of lumbar TENS on perceived exertion, ventilatory pattern and muscle oxygenation. We hypothesised that endurance capacity would be improved with lumbar TENS due to a blunted response in exercise ventilation, which would contribute to improve ventilatory efficacy and reduce exercise dyspnoea. Conversely, we hypothesised that any mitigation in cardiac output (if any) would be compensated by an increase in peripheral muscular oxygen extraction.
\end{abstract}

Consecutive subjects with severe stable COPD referred for pulmonary rehabilitation were screened for eligibility between October 2017 and October 2018. Inclusion criteria were patients aged $\geqslant 18$ years and naive to TENS. Non-inclusion criteria were contraindication to TENS, opiate treatment during the previous 3 months and pregnancy. 25 patients were screened; 12 did not meet the inclusion criteria, two declined to participate, one had recently used opiate treatment and 10 agreed to participate (and provided signed consent; approved by the French ethics committee Est III (number 17.05.15)), which was the sample size chosen to gather preliminary data. Included patients performed three constant workload exercise testing (CWET; 75\% of the maximal workload achieved during a previously performed incremental cardiopulmonary exercise testing (CPET)) up to exhaustion, with $\geqslant 24$-h rest period between tests, with either high-frequency $(100 \mathrm{~Hz}, 100 \mu \mathrm{s})$, low-frequency $(4 \mathrm{~Hz}, 100 \mu \mathrm{s})$ or sham $(100 \mathrm{~Hz}, 100 \mu \mathrm{s}$, intermittent placebo [6]) TENS in a randomised order (computer-generated sequence and concealed allocation). TENS was set at rest, $10 \mathrm{~min}$ prior to each CWET, and maintained throughout the test. The current was biphasic, symmetric and applied through four self-adhesive surface electrodes $(5 \times 5 \mathrm{~cm})$ positioned by pair at the L3-L4 level, $2 \mathrm{~cm}$ lateral to the lower border of the corresponding vertebrae.

@ERSpublications

Lumbar transcutaneous electrical nerve stimulation aimed to block muscle group III-IV sensory afferents does not improve endurance exercise capacity in patients with COPD http://bit.ly/2lZr6Mt

Cite this article as: Bonnevie T, Gravier F-E, Prieur G, et al. Lumbar transcutaneous electrical nerve stimulation to improve exercise performance in COPD patients. Eur Respir J 2019; 54: 1900784 [https:// doi.org/10.1183/13993003.00784-2019]. 
TABLE 1 Impact of lumbar transcutaneous electrical nerve stimulation (TENS) on endurance exercise capacity and physiological parameters measured at isotime and time limit

\section{TENS settings}

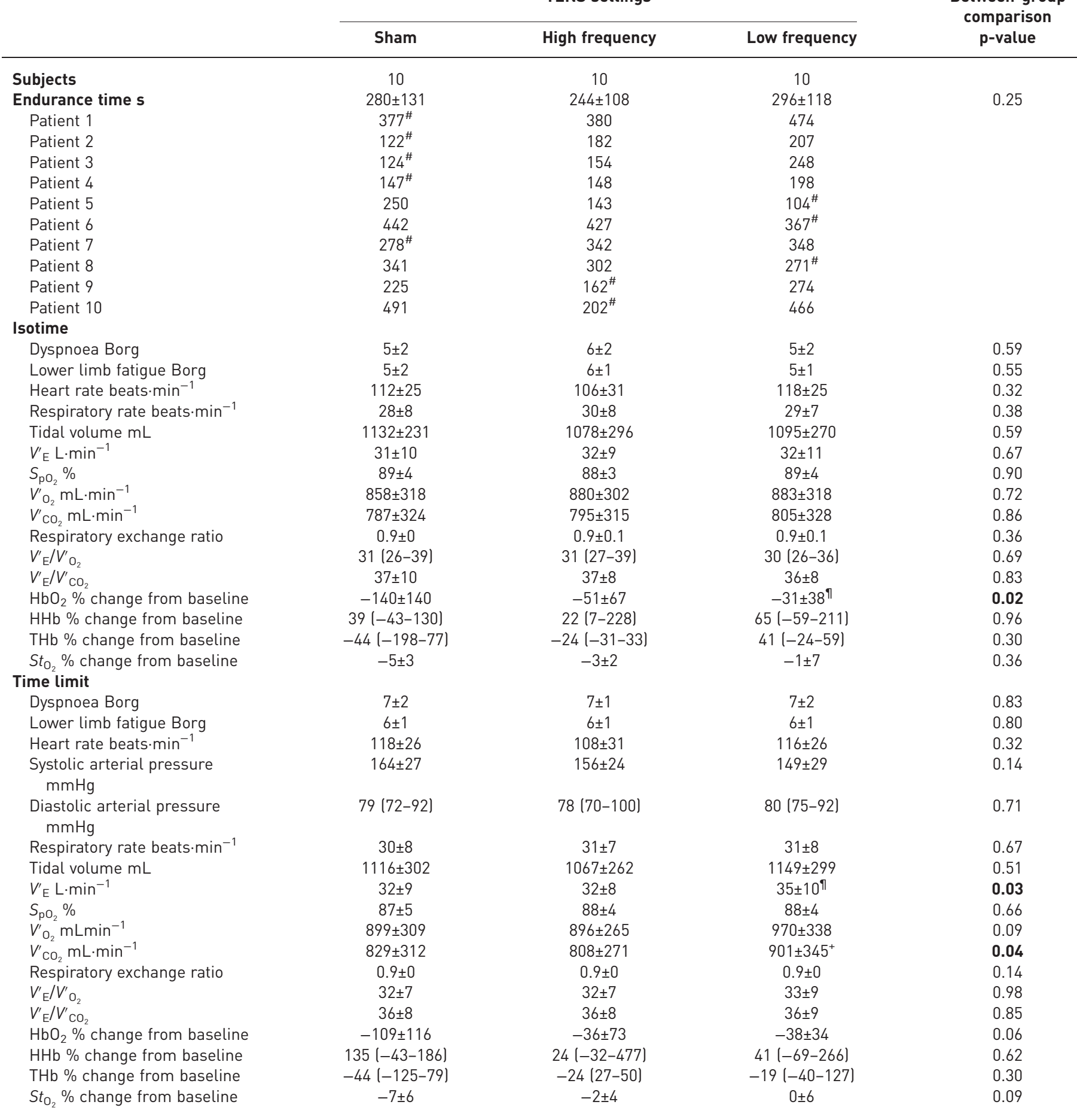

Data are presented as $\mathrm{n}$, mean $\pm \mathrm{SD}$ or median (interquartile range), unless otherwise stated. Repeated measures of ANOVA or Friedman test according to the distribution. $V_{\mathrm{E}}^{\prime}$ : minute ventilation; $\mathrm{S}_{\mathrm{PO}_{2}}$ : oxygen saturation measured by pulse oximetry; $V^{\prime}{ }_{2}$ : oxygen consumption; $V^{\prime} \mathrm{CO}_{2}$ : carbon dioxide production; $\mathrm{HbO}_{2}$ : oxyhaemoglobin and oxymyoglobin; $\mathrm{HHb}$ : deoxyhaemoglobin and deoxymyoglobin; THb: total haemoglobin and myoglobin; $\mathrm{St}_{\mathrm{O}_{2}}$ : muscle tissue oxygen saturation. \#: time limit of the shortest constant workload exercise testing used to define isotime for the subsequent analysis; " ": Tukey's post hoc comparison, significantly higher than sham TENS, $\mathrm{p}<0.05 ;^{+}$: Tukey's post hoc comparison, significantly higher than high-frequency TENS, $p<0.05$. 
The intensity was adjusted just under the pain threshold every $3 \mathrm{~min}$ (and no longer increased during the CWET). Patients were told that they may no longer feel the current during the procedure due to accommodation (or the intermittent nature of the sham TENS), but were asked not to discuss their bodily sensations in order to maintain study blinding. Perceived exertion (dyspnoea and lower limb fatigue assessed every $30 \mathrm{~s}$ using the Borg scale), gas exchange and ventilatory patterns (including oxygen consumption and carbon dioxide production, recorded breath by breath (Vyntus CPX; Care Fusion, San Diego, CA, USA) and muscle oxygenation (vastus lateralis muscle relative change in total haemoglobin and myoglobin (THb), oxyhaemoglobin and oxymyoglobin $\left(\mathrm{HbO}_{2}\right)$ and deoxyhaemoglobin and deoxymyoglobin), continuously recorded at a frequency of $1 \mathrm{~Hz}$ using a near-infrared spectroscopy device (NIRS; PortaMon; Artinis, Einsteinweg, the Netherlands) and tissue oxygen saturation $\left(S_{\mathrm{tO}_{2}}\right)$, calculated as the ratio of $\mathrm{HbO}_{2} / \mathrm{THb} \times 100$ and expressed as change from baseline were monitored during the tests. Statistical analysis was performed using Prism 5 software (GraphPad, San Diego, CA, USA) and comparison between tests was performed using either repeated ANOVA and Tukey post hoc tests or Friedman and Wilcoxon post hoc tests for pairwise comparison according to the distribution. Secondary outcomes were analysed both at time limit $\left(t_{\lim }\right)$ and at isotime. Isotime was defined as the $t_{\lim }$ of the shortest CWET.

Patients were aged 64 (interquartile range (IQR) 57-67) years, had severe bronchial obstruction (mean \pm SD forced expiratory volume in $1 \mathrm{~s} 0.9 \pm 0.3 \mathrm{~L}$ ), significant thoracic distension and decreased exercise capacity (median oxygen uptake 12.3 (IQR $11.2-18.7) \mathrm{mL} \cdot \mathrm{kg}^{-1} \cdot \mathrm{min}^{-1}$ ). They all had a ventilatory limitation during the initial CPET. There was no significant difference in endurance capacity between low-frequency, high-frequency and sham TENS nor in dyspnoea or lower limb fatigue at isotime or $t_{\lim }$ (table 1). Gas exchange and ventilatory patterns during exercise are shown in table 1. NIRS data were not available in three patients, due to technical reasons. $\mathrm{HbO}_{2}$ was significantly different between low-frequency, high-frequency and sham TENS at isotime, mainly due to a significant difference between low-frequency and sham TENS $(\mathrm{p}<0.05)$. A similar trend was observed at $t_{\lim }(\mathrm{p}=0.06)$. Finally, a trend toward an improved $S_{\mathrm{tO}_{2}}$ for low-frequency TENS was also observed at $t_{\text {lim }}$ (table 1).

Our study did not show any improvement in endurance exercise capacity with either high-frequency or low-frequency TENS. Overall metabolism and exercise ventilation tended to increase with low-frequency TENS while NIRS revealed that local rectus femoris oxygenation tended to improve with both currents. The lack of effects observed with high-frequency TENS may be related to an activation of specific opioid receptor. While both the fentanyl and low-frequency TENS activate the same $\mu$-opioid receptors, high-frequency TENS effects are mediated through the $\delta$-opioid receptor [4]. NIRS data are frequently used as an indirect surrogate for muscular oxygenation [7]. Therefore, the present results suggest that TENS might improve the ratio between local oxygen delivery and consumption. Among potential mechanisms, an improvement in local oxygen supply through a TENS-mediated sympathovagal modulation may improve vascular conductance and local blood flow [8]. Alternatively, TENS may have change spatial recruitment (out of the range of the superficial NIRS area of interrogation) or the type of muscle fibres recruitment during exercise [9]. A shift toward the recruitment of fast twitch muscular fibres (type II), which are energetically less efficient, would contribute to explain the concomitant increase in metabolism observed with low-frequency TENS [10].

In conclusion, this study does not provide substantive clinical or physiological argument for a positive effect of lumbar TENS on exercise endurance capacity in stable COPD patients.

Tristan Bonnevie $\oplus^{1,2}$, Francis-Edouard Gravier $\oplus^{1,2}$, Guillaume Prieur ${ }^{2,3}$, Yann Combret $\oplus^{4,5}$, David Debeaumont ${ }^{6}$, Maxime Patout ${ }^{2,7}$, Bouchra Lamia ${ }^{2,3,7}$, Jean-François Muir ${ }^{1,2,7}$, Clement Médrinal ${ }^{2,3}$ and Antoine Cuvelier $\overbrace{}^{2,7}$

${ }^{1}$ ADIR Association, Rouen University Hospital, Rouen, France. ${ }^{2}$ Normandie University, UNIROUEN, UPRES EA 3830, Rouen University Hospital, Haute Normandie Research and Biomedical Innovation, Rouen, France. ${ }^{3}$ Intensive Care Unit Dept, Le Havre Hospital, Le Havre, France. ${ }^{4}$ Physiotherapy Dept, Le Havre Hospital, Le Havre, France. ${ }^{5}$ Institute of Experimental and Clinical Research (IREC), Pole of Pulmonology, ORL and Dermatology, Catholic University of Louvain, Brussels, Belgium. ${ }^{6}$ Dept of Respiratory and Exercise Physiology and CIC-CRB 1404, Rouen University Hospital, Rouen, France. ${ }^{7}$ Pulmonary, Thoracic Oncology and Respiratory Intensive Care Dept, Rouen University Hospital, Rouen, France.

Correspondence: Tristan Bonnevie, ADIR Association, 147 Avenue du Maréchal Juin, 76230 Bois-Guillaume, France. E-mail: rehabilitation@adir-hautenormandie.com

Received: 18 April 2019 | Accepted after revision: 29 Aug 2019

The protocol was prospectively registered on www.clinicaltrials.gov (NCT03312322). Data will be shared on request to the corresponding author, for the purposes of meta-analyses.

Conflict of interest: T. Bonnevie has nothing to disclose. F-E. Gravier has nothing to disclose. G. Prieur has nothing to disclose. Y. Combret has nothing to disclose. D. Debeaumont has nothing to disclose. M. Patout reports grants from 
B\&D Electromedical and ADIR Association, personal fees from ResMed and Philips Respironics, grants and non-financial support from Fisher \& Paykel, non-financial support from MSD and Asten, during the conduct of the study. B. Lamia has nothing to disclose. J-F. Muir has nothing to disclose. C. Médrinal has nothing to disclose. A. Cuvelier has nothing to disclose.

\section{References}

1 Amann M, Blain GM, Proctor LT, et al. Group III and IV muscle afferents contribute to ventilatory and cardiovascular response to rhythmic exercise in humans. J Appl Physiol 2010; 109: 966-976.

2 Amann M, Blain GM, Proctor LT, et al. Implications of group III and IV muscle afferents for high-intensity endurance exercise performance in humans. J Physiol 2011; 589: 5299-5309.

3 Gagnon P, Bussières JS, Ribeiro F, et al. Influences of spinal anesthesia on exercise tolerance in patients with chronic obstructive pulmonary disease. Am J Respir Crit Care Med 2012; 186: 606-615.

4 Kalra A, Urban MO, Sluka KA. Blockade of opioid receptors in rostral ventral medulla prevents antihyperalgesia produced by transcutaneous electrical nerve stimulation (TENS). J Pharmacol Exp Ther 2001; 298: 257-263.

5 Sluka KA, Deacon M, Stibal A, et al. Spinal blockade of opioid receptors prevents the analgesia produced by TENS in arthritic rats. J Pharmacol Exp Ther 1999; 289: 840-846.

6 Rakel B, Cooper N, Adams HJ, et al. A new transient sham TENS device allows for investigator blinding while delivering a true placebo treatment. J Pain 2010; 11: 230-238.

7 Van Beekvelt MC, Colier WN, Wevers RA, et al. Performance of near-infrared spectroscopy in measuring local $\mathrm{O}_{2}$ consumption and blood flow in skeletal muscle. J Appl Physiol 2001; 90: 511-519.

8 Vieira PJ, Ribeiro JP, Cipriano G Jr, et al. Effect of transcutaneous electrical nerve stimulation on muscle metaboreflex in healthy young and older subjects. Eur J Appl Physiol 2012; 112: 1327-1334.

9 Tomasi FP, Chiappa G, Maldaner da Silva V, et al. Transcutaneous electrical nerve stimulation improves exercise tolerance in healthy subjects. Int J Sports Med 2015; 36: 661-665.

10 Coyle EF, Sidossis LS, Horowitz JF, et al. Cycling efficiency is related to the percentage of type I muscle fibers. Med Sci Sports Exerc 1992; 24: 782-788. 